\title{
High Resolution Characterization of Bioengineered Tissue using Multiphoton Microscopy.
}

\author{
W.R. Zipfel,* R.D. Bowles, * L.J. Bonassar*** and R.M. Williams*
}

*Department of Biomedical Engineering, Cornell University, Ithaca, NY 14853

**Sibley School of Mechanical and Aerospace Engineering, Cornell University, Ithaca, NY 14853

Although a number of different imaging modalities are available for characterizing bioengineered materials and tissues, the cellular-level resolution and molecular specificity of optical microscopy methods are often required for many studies. However, bioengineered tissues are often 3D structures where conventional widefield microscopy is of little use and the ability to optically section is critical. To achieve optical sectioning, confocal microscopy is often employed, which uses a pinhole to reject out-of-focus fluorescence. Depending on the optical properties of the structure, confocal microscopy can provide 3D imaging to depths greater than $100 \mu \mathrm{m}$, but in the end is always limited by scattered out-of-focus background fluorescence since imaging of the emitted light is required. Multiphoton microscopy (MPM), based on nonlinear absorption and harmonic generation (nonlinear scattering), achieves optical sectioning in a fundamentally different way. The nonlinear scaling of excitation probability creates a 3D localized observation volume [1] and is capable of acquiring signal at larger focal depths since even the scattered fluorescence arises from the focal volume. This deep sectioning ability has made multiphoton microscopy the method of choice for fluorescence based in vivo imaging, and combined with MPM's unique ability to directly image unlabeled collagen by second harmonic generation (SHG) [2], this form of laser scanning microscopy is ideal for the high resolution imaging, characterization and quantification of bioengineered materials and tissues.

A major advantage of MPM for studies of engineered tissues lies in its ability to yield highresolution images of collagen structure. Because collagen is chiral, it contains anharmonic electronic normal modes that yield a strong SHG radiation field. On the micron spatial scale, SHG imaging provides a direct look at collagen fibril formation and orientation. This can be used, for example, to monitor the microscopic structure of engineered tissues as it forms under different bioreactor conditions [3] (Fig. 1A, B). More quantitative measures of fibril orientation include FFT based analysis which can return average orientation (Fig. 1C, D). Collection of SHG emissions in the transmitted direction, in addition to normal epi-collection, can provide additional detailed information on the 3D structure and fibril sizes in tissue constructs. Based on calculations from nonlinear scattering theory under tightly focused light [4], an understanding of the magnitude and directionality dependence of SHG emission can be modeled (Fig. 2A) and experimentally demonstrated (Fig. 2B, C). This information can be used to elucidate collagen structures at the $\mathrm{nm}$ level in natural tissues, such as tendon [5] and in bioengineered tissue constructs.

\section{References}

[1] W. Denk et al., Science. 248 (1990) 73.

[2] W.R. Zipfel et al., PNAS. 100 (2003)

[3] R.D. Bowles et al., Tissue Engineering Part A. (2010) doi:10.1089/ten.tea.2009.0442.

[4] L. Moreaux et al., J. Opt. Soc. Am. B 17 (2000) 1685.

[5] R. M. Williams et al., Biophys. J. 88 (2005) 1377. 


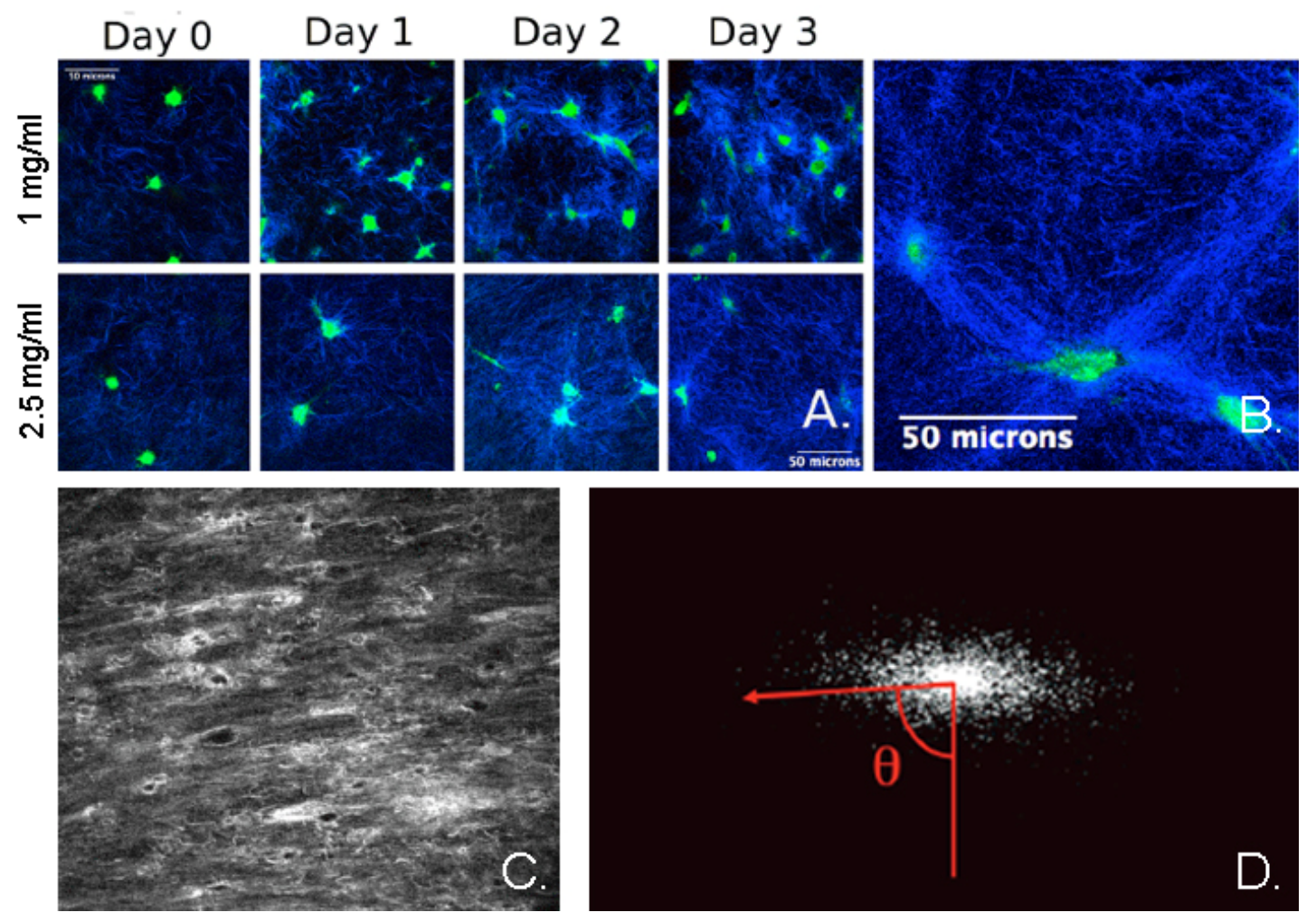

FIG. 1. SHG/MPM fluorescence images of tissue engineered annulus fibrosus (outer portion of the intervertebral disc) created using a collagen gel contraction method. A. Images from inside annulus region during contraction of $1 \mathrm{mg} / \mathrm{ml}$ and $2.5 \mathrm{mg} / \mathrm{ml}$ collagen disk constructs over 3 days (B.) and magnified image showing aligned fibers between cells on Day 3 of contraction in $2.5 \mathrm{mg} / \mathrm{ml}$ collagen disks (blue - collagen, green - cell). C and D: SHG image of engineered annulus fibrosus and FFT analysis to obtain the average fibril orientation (D).
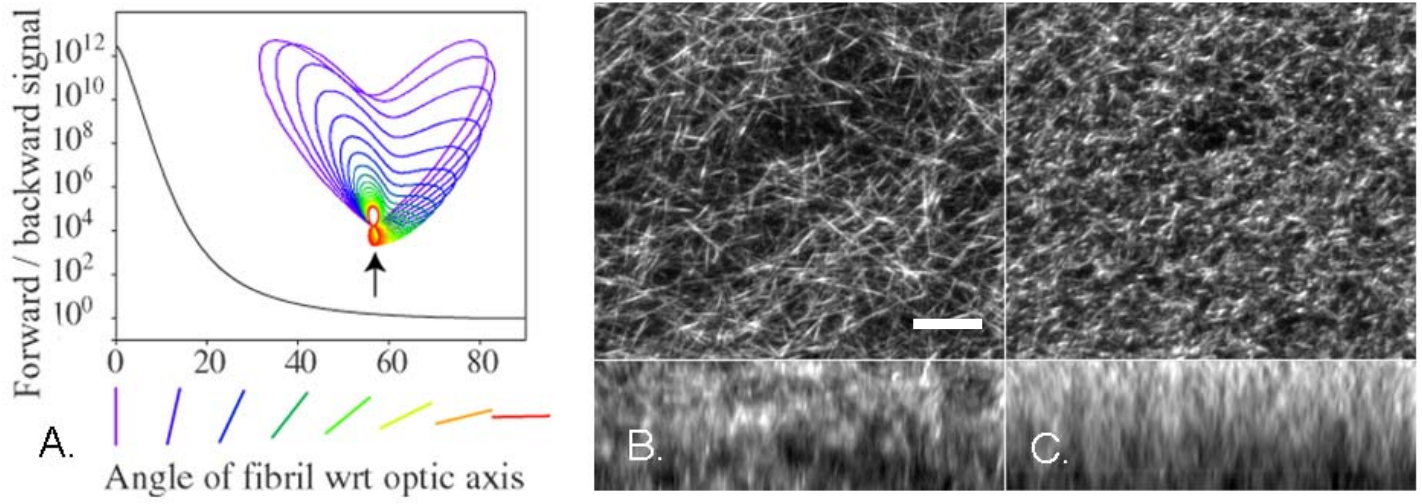

FIG. 2. Directionally of SHG vs. fibril orientation. A. Forward to backward emitted SHG as a function of fibril angle to the optical axis; insert: color-coded SHG intensity profiles from the variously oriented rods diagramed under the $\mathrm{X}$ axis. B. Backward (epi) collected SHG image of a $\sim 10$ um thick collagen gel (lateral projection - top; axial projection - bottom; scale bar: $10 \mathrm{um}$ ). C. Simultaneously acquired forward directed SHG signal. B and C verify the calculations shown in the plot in A. 